\title{
Compact Broadband Terahertz Perfect Absorber based on Multi-interference and Diffraction Effects
}

\author{
Cheng Shi ${ }^{1,2}$, XiaoFei Zang ${ }^{1 *}$, Lin Chen ${ }^{1}$, Yan Peng ${ }^{1}$, Bin Cai ${ }^{1}$,Geoffery Nash ${ }^{2}$ and YiMing Zhu ${ }^{1 *}$
}

\begin{abstract}
High absorption over a wide frequency band has attracted considerable interest due to its potential applications in imaging and anti-radar cloaking devices. In order to make these devices more integrated, the thickness of the absorber is crucial. In this letter, a compact, polarization-independent, broadband, omnidirectional terahertz ( $\mathrm{THz}$ ) absorber is proposed, fabricated, and evaluated. This THz absorber is based on the combined effect of the multi-interference and diffraction. It is experimentally demonstrated that over $95 \%$ absorption can be obtained in the frequency range from $0.75 \mathrm{THz}$ to $2.41 \mathrm{THz}$. This well-designed thinner absorber not only reduces the device thickness to $120 \mu \mathrm{m}$, but also introduces more interference peaks so that the absorption spectrum is significantly enlarged. Five successive absorption peaks at $0.88 \mathrm{THz}, 1.20 \mathrm{THz}, 1.53 \mathrm{THz}, 1.96 \mathrm{THz}$, and $2.23 \mathrm{THz}$ are combined into a broadband THz absorption spectrum.
\end{abstract}

Index Terms- Metamaterial, Terahertz absorber, multi-interference

\section{INTRODUCTION}

$\mathrm{T}$ HE concept of trapping energy over a wide bandwidth has been one of the hot research topics for decades. To date, these energy trapping devices have found significant applications in diverse areas including sensor detection, ${ }^{1}$ power harvesting, ${ }^{2}$ imaging spectrum system, ${ }^{3}$ and so on. With the advent of metamaterials, nearly unity power can be captured at a certain frequency. The metamaterial perfect absorber (MPA) was first reported in 2008, ${ }^{4,5}$ which consists of a three-layer structure that includes a subwavelength frequency selective

Manuscript received

; revised

; accepted

This work was partly supported by National Program on Key Basic Research Project of China (973 Program, 2014CB339806), Basic Research Key Project (12JC1407100), Major National Development Project of Scientific Instrument and Equipment (2011YQ150021) (2012YQ14000504), National Natural Science Foundation of China (11174207) (61138001) (61205094) (61307126), Shanghai Rising-Star Program (14QA1403100), Program of Shanghai Subject Chief Scientist (14XD1403000), the Scientific Research Innovation Project of Shanghai Municipal Education Commission (14YZ093), and the New Century Excellent Talents Project from Ministry of Education (NCET-12-1052).

1.The authors are with the Shanghai Key Lab of Modern Optical System and Engineering Research Center of Optical Instrument and System, Ministry of Education, University of Shanghai for Science and Technology, Shanghai 200093, China (e-mail: xdzang@usst.edu.cn, ymzhu@usst.edu.cn).

2.The authors are with the EPSRC Center of Doctoral Training in Metamaterials $\left(\mathrm{XM}^{2}\right)$, University of Exeter, Exeter, Devon, EX4 4QL, United Kingdom.

Color versions of one or more of the figures in this paper are available online at http://ieeexplore.ieee.org.

Digital Object Identifier: surface (FSS) layer, a dielectric spacer layer, and a metal ground plane. The electric ring resonator (ERR) in the FSS layer can introduce a phase change at a given frequency, which leads to the destructive interference of the reflective waves and results in an absorption peak. ${ }^{6,7}$ Obviously, a single electric ring resonator (ERR) structure in the FSS can lead to only one resonance frequency in the absorption spectrum. ${ }^{8}$ To realize a broadband absorption spectrum, one must design several FSS layers with slightly different ERR structures and spacer layers with different thicknesses to make the absorption peaks closely positioned. By stacking the well-designed FSS/dielectric layers, these absorption peaks can be merged into a broadband absorption spectrum. ${ }^{9,10}$ However, stacking layers increases the difficulty of fabrication and makes it hard for such terahertz absorbers $^{11}$ to realize the expected performances in the experiments. $^{12}$ Recently, heavily-doped silicon grating terahertz absorbers ${ }^{13,14,15}$ have been reported as an alternative design for achieving high absorption for a broad bandwidth in the terahertz regime. Since the diffractions of different orders can reduce the reflection at certain frequencies, the grating structure is designed to eliminate the reflection of the materials (i.e. heavily doped silicon). The grating absorbers can substantially simplify the fabrication of the broadband absorber. At the meantime, it requires a thick substrate to passively absorb the diffracted power. However, the interference caused by the upper and lower interface of the grating has a large free spectral range (FSR). The large spectral range between two interference peaks limited the absorption bandwidth, because only one interference peak can be combined with the diffraction peaks to form the absorption spectrum (Fig. 1(a)). In this letter, a new design is proposed and demonstrated which can not only reduce the thickness of the broadband absorber but also merge more absorption peaks into an even broader absorption spectrum (Fig. 1(b)).

Our new design develops new features of the substrate layer and also decreases its thickness. By reducing the substrate's thickness and depositing a thin metallic film on the back side of the substrate, where the substrate layer acts as a dispersive resonant cavity instead of a lossy material which reduces the transmitted energy passively. By combining the advantages of metamaterial absorbers and grating absorbers, we exploit simultaneously multi-interference effect and diffraction effect. The reflected waves from the upper and lower interface of the substrate will interference with each other. The destructive 
interferences of the reflected waves form the peaks while the constructive interferences of the reflected waves form the dips in the absorption spectrum (blue line in Fig. 1(b)). We then carefully design the grating structure so that the enhanced reflection related to the constructive interference can be eliminated by antireflection caused by the diffraction of the gratings. With a proper design, these two effects can be combined with each other and form a broadband absorption spectrum. Our design substantially enlarges the bandwidth of the absorption spectrum as well as simplifies the fabrication, while such a broadband absorption spectrum combined by five absorption peaks usually requires much thicker multi-layer grating absorbers ${ }^{16}$ or more complicated stacked MPAs. Our $\mathrm{THz}$ absorber presents over $90 \%$ absorption within the range from $0.65 \mathrm{THz}$ to $2.45 \mathrm{THz}$. The relative absorption bandwidth (RAB) reaches up to $120 \%$, broader than any other absorber in this frequency range to the best of our knowledge. ${ }^{17}$

\section{METHOD}

\section{A. Multi-interference Model}

The absorber is made up of three layers, which is similar to the construction of the single-resonance MPA. In our design, the traditional metal ERR and the polyimide spacer are replaced by the heavily-doped silicon grating and spacer (Figs. 1(c) and 1(d)). The geometric parameters are as follows: $w=22 \mu \mathrm{m}, l=$ $73 \mu \mathrm{m}, p=105 \mu \mathrm{m}, t_{1}=51 \mu \mathrm{m}, t_{2}=74 \mu \mathrm{m}, t_{3}=200 \mathrm{~nm}$. As the thickness of the metal plate is much larger than the typical skin depth in the terahertz regime, the system absorption is only affected by the reflection in the grating surface. To analyze how the thinner substrate acts as the resonant cavity, the interference model is established as illustrated in Fig. 2(a). The reflection waves of different orders can be calculated as follows:

$$
\begin{aligned}
& \widetilde{I_{0}}=I_{0} e^{i w t} \\
& \widetilde{r_{0}}=r_{11} I_{0} e^{i\left(w t+\theta_{11}\right)} \\
& \tilde{r_{1}}=A^{2} t_{12} t_{21} I_{0} e^{i\left(w t+\theta_{12}+\theta_{21}+2 \delta+\pi\right)}, \\
& \tilde{r_{2}}=A^{2} r_{22} e^{i\left(\theta_{22}+2 \delta+\pi\right)} \tilde{r}_{1}=q \tilde{r}_{1} \\
& \tilde{r_{n}}=q^{n-1} \tilde{r_{1}}
\end{aligned}
$$

where $I_{0}$ is the magnitude of the incident wave, $r_{i i}, \theta_{i i}$ are the magnitude and phase coefficients for the reflected waves from $i^{\text {th }}$ layer back to $i^{\text {th }}$ layer, $t_{i j}, \theta_{i j}$ are the magnitude and phase coefficients for the transmitted waves from $i^{\text {th }}$ layer to $j^{\text {th }}$ layer, $A=e^{-\frac{2 \pi}{\lambda} k d}, \delta=n k_{0} d$ are the propagation phase and loss caused by spacer layer with a thickness of $d$ and refractive index $\tilde{n}=n-i \kappa$. These coefficients at individual interfaces can be derived from numerical simulations by decoupling the model (Figs. 2(b) and 2(c)). ${ }^{19}$ In this work, by optimizing the doping density, we chose $0.54 \Omega \mathrm{cm}$ boron-doped silicon, whose permittivity can be expressed by Drude model as: ${ }^{19}$ $\varepsilon(\omega)=11.7-\frac{3.64 \times 10^{26}}{\omega^{2}+1.74 \times 10^{13} \omega i}$.

The high value of the imaginary part of the permittivity can lead to a relative large $A$. As the thickness of the substrate increases, the reflection waves of higher orders decline exponentially. In the aforementioned grating absorber, the substrate is so thick that all other reflective waves except $\widetilde{r_{0}}$ tend to zero, so the role of the substrate is only to absorb the transmitted energy. We optimize the thickness of the substrate to a certain value, which will make the reflection waves of different orders interference with each other. The thinner spacer layer will now be evolved to a resonant cavity and introduce more interference. We can change the FSR to manipulate the frequencies of the interference positioned in the spectrum by adjusting the thickness of the substrate. In our case, the thickness of substrate $d$ is chosen as $74 \mu \mathrm{m}$, the magnitude of $\widetilde{r_{2}}$ will be less than $0.01 I_{0}$, which makes the overall reflection waves are mainly contributed by $\widetilde{r}_{0}$ and $\tilde{r}_{1}$. From (1), we can conclude that when $\theta_{12}+\theta_{21}+2 \delta+\pi-\theta_{22}=2 m \pi+\pi(\mathrm{m} \in \mathrm{Z})$, the overall reflection will reach the minima at $f_{1}=0.87 \mathrm{THz}, f_{3}=$ $1.54 \mathrm{THz}$ and $f_{5}=2.25 \mathrm{THz}$, since $\widetilde{r}_{0}$ and $\tilde{r}_{1}$ will destructively interference with each other (black dash lines in Figs. 2(d) and 2(f)).

\section{B. Impedance-matching Condition of Diffraction Gratings}

In order to achieve a broadband anti-reflection spectrum, enhanced reflection related to the constructive interference has to be tailored. Recent research has shown the grating on the heavily-doped silicon can reduce the reflection at certain frequencies. ${ }^{17}$ Thus the diffraction grating structure is applied to eliminate the high reflection caused by the constructive interference. The impedance-matching condition will be fulfilled when the impedance of the grating is ${ }^{16}$ $Z_{\text {gra }}=Z_{0} \sqrt{\cos (\phi) / n}$, where $\phi=\sin ^{-1}(m \lambda /(n p))$ is the diffraction angle of the $\mathrm{m}^{\text {th }}$ order diffraction and $n$ is the refractive index of the silicon spacer. Through the two dimensional rigorous coupled wave analysis (2D-RCWA) method, ${ }^{20}$ the diffraction efficiency of different orders is calculated, as demonstrated in Fig. 3(a). The gray zone in Fig. 3 indicts the frequency band where the $[ \pm 1,0]$ order diffraction is dominant. The ideal impedance of the grating should be the combination of the $[0,0]$ order-impedance in the $[0,0]$ order diffraction zone (red solid line in the white zone in Fig. 3(b)) and the $[ \pm 1,0]$ order-impedance in the $[ \pm 1,0]$ order diffraction zone (blue solid line in the gray zone in Fig. 3(b)). We retrieve the effective impedance of the grating by adopting the S-parameter retrieval method ${ }^{21}$ and optimize the parameters of the grating to make its impedance equal to the ideal impedance at the corresponding frequencies. Based on the optimization, the impedance-matching condition is thus reached at $f_{2}=1.16$ $\mathrm{THz}, f_{3}=1.54 \mathrm{THz}$ and $f_{4}=1.95 \mathrm{THz}$ (highlighted as orange in Figs. 3(b) and 3(c)). The distributions of the electric field at 
these frequencies (Figs. 3(d), 3(e) and 3(f)) illustrate the strong $[0,0]$-order diffraction at $f_{2}=1.16 \mathrm{THz}$, and $[ \pm 1,0]$-order diffraction at $f_{3}=1.54 \mathrm{THz}$ and $f_{4}=1.95 \mathrm{THz}$, which supports our analysis. So it can be concluded that the successive reflection dips of the absorber in a wide frequency region (Fig. 2(f)) results from the impedance-matching condition of the diffraction of the grating superposed on the destructive interference of the multi-layer structure (black and red dash line in Figs. 2(d), 2(e) and 2(f)) .

\section{EXPERIMENTS}

To verify this analysis, the theoretical absorbance of designed absorber with two pairs of perfect electric walls and perfect magnetic walls bounding a propagation region was simulated via CST microwave studio ${ }^{\circledR}$. As illustrated in Fig. 4(a), five near-unity absorption peaks can be observed in the simulated absorption spectrum which has a good agreement with our analysis. Furthermore, the energy density distributions of the absorber at each absorption peak frequency are plotted in Figs. 4(b)-(f), which clearly reveal that the energy is concentrated at the grating/spacer interface at $f_{2}, f_{3}, f_{4}$ and the energy are captured in the spacer at $f_{1}, f_{3}, f_{5}$ due to the destructive interference. These distributions confirm our analysis on how the destructive interference and the impedance-matching condition of the grating contribute to the absorption peaks.

Standard wafer grinding, metal evaporation, optical lithography, and inductively coupled plasma (ICP) etching techniques were used to fabricate the multilayer absorber. First, a $500 \mu$ m-thick silicon wafer was grinded to a thickness of 120 $\mu \mathrm{m}$. A $20 / 180 \mathrm{~nm} \mathrm{Ti} / \mathrm{Au}$ metallic plate was then evaporated onto the silicon wafer. On the other side of the wafer, the grating structure was formed by the optical lithography of the photo resist and ICP etching.

We used a terahertz time domain spectroscopy (THz-TDS) system $^{22}$ to experimentally evaluate the behavior of the fabricated absorber by measuring the reflectance spectra. The THz-TDS system can produce terahertz waves in the range of $0.2-2.8 \mathrm{THz}$ with an $8 \mathrm{GHz}$ spectral resolution. The measured spectra in transverse electric (TE) and transverse magnetic (TM) mode (Figs. 5(a) and 5(b)) reveal five absorption peaks at the frequency $0.88 \mathrm{THz}, 1.20 \mathrm{THz}, 1.53 \mathrm{THz}, 1.96 \mathrm{THz}$ and 2.23 $\mathrm{THz}$ with absorbance of 99.7\%, 95.8\%, 99.9\%, 99.5\%, and $99.9 \%$, respectively. Due to these five closely positioned absorption peaks, a wide frequency band is achieved from 0.75 THz to $2.41 \mathrm{THz}$, where the absorption is greater than 95\%. Excellent agreement between simulation and experiment spectra is observed, where the slight differences can be attributed to, which may be caused by the tolerance of the samples fabrication and measurement errors.

\section{Discussion}

Numerical simulations of absorption spectrum with different angles of incidence under TE and TM polarization state are illustrated in Figs. 5(c) and 5(d). For obliquely incident wave of TE and TM mode, the $x$ component of the incident electric field in TE mode and incident magnetic field in TM mode will decrease with the increase of the angles of incidence. This will change the impedance of the structure which will affect the $2^{\text {nd }}$, $3^{\text {rd }}, 4^{\text {th }}$ peak. However, since the 1 st, 5 th peaks are mainly caused by the interference effect and irrelevant to the impedance, only slight differences and blue shifts of these peaks in both modes can be observed (the white dash lines in Figs 5(c) and 5(d)). The absorber can still maintain an over 90\% absorbance at a large incidence angle ranges from 0 degree to 50 degree under both polarization states. Furthermore, owing to the symmetrical design of the device, the absorption response is insensitive to polarizations states of the normal incident wave.

\section{CONCLUSION}

In summary, we have theoretically purposed and experimentally demonstrated a nearly perfect broadband compact absorber in $\mathrm{THz}$ regime. The absorber is much simpler to fabricate and has a larger RAB than other broadband MPA in the $\mathrm{THz}$ frequency range. The fabricated absorber presents $>95 \%$ absorption over a frequency range of $1.7 \mathrm{THz}$. Destructive interference theory and impedance-matching condition of the grating are applied to elucidate the five successive absorption peaks at $0.88 \mathrm{THz}, 1.20 \mathrm{THz}, 1.53 \mathrm{THz}, 1.96 \mathrm{THz}$, and 2.23 THz. Owing to the symmetrical design, the absorber is also insensitive to the polarization states of the incident wave. Furthermore, the absorber is also valid to a wide range of incident angles for both TE and TM polarizations.

\section{REFERENCES}

[1] Liu, N., Mesch, M., Weiss, T., Hentschel, M. \& Giessen, H. Infrared perfect absorber and its application as plasmonic sensor controlling electromagnetic fields. Nano Lett. 10, 2342-2348 (2010).

[2] Ferry, V. E. et al. Light trapping in ultrathin plasmonic solar cells. Optics Express 18, A237-A245 (2010).

[3] Liu, X., Starr, T., Starr, A. F. \& Padilla, W. J. Infrared spatial and frequency selective metamaterial with near-unity absorbance. Phys. Rev. Lett. 104, 207403 (2010).

[4] Landy, N. I., Sajuyigbe, S., Mock, J. J., Smith, D. R. \& Padilla, W. J. Perfect Metamaterial absorber. Phys. Rev. Lett. 100, 207402 (2008).

[5] Tao, H. et al. A metamaterial absorber for the terahertz regime: design, fabrication and characterization. Opt. Express 16, 7181-7188 (2008).

[6] Chen, H. T. Interference theory of metamaterial absorber. Opt. Express 20, 7165-7172 (2012).

[7] Chen, H. T. et al. Antireflection coating using metamaterials and identification of its mechanism. Phys. Rev. Lett. 105, 073901-4 (2010).

[8] Hokmabadi, M. P., Wilbert, D. S., Kung, P. \& Kim, S. M. Terahertz metamaterial absorbers. Terahertz Science and Technology 6, 40-58 (2013).

[9] Wang, B. X. et al. A simple design of ultra-broadband and polarization insensitive terahertz metamaterial absorber. Applied Physics A 115, 1187-1192 (2014).

[10] Ye, Y. Q., Jin, Y. \& He, S. Omnidirectional, polarization-insensitive and broadband thin absorber in the terahertz regime. J. Opt. Soc. Am. B 27, 498-504 (2010).

[11] Cui, Y. et al. Ultrabroadband light absorption by a sawtooth anisotropic metamaterial slab. Nano Lett. 12, 1443-1447 (2012).

[12] Grant, J., Ma, Y., Saha, S., Khalid, A. \& Cumming, D. R. Polarization insensitive, broadband terahertz metamaterial absorber. Opt. Lett. 36, 3476-3478 (2011). 
[13] Shi, C. et al. A polarization-independent broadband terahertz absorber. Appl. Phys. Lett. 105, 031104 (2014).

[14] Pu, M. et al. Engineering heavily doped silicon for broadband absorber in the terahertz regime. Opt. Express 20, 25513-25519 (2012).

[15] Kakimi, R., Fujita, M., Nagai, M., Ashida, M. \& Nagatsuma. T. Capture of a terahertz wave in a photonic-crystal slab. Nat. Photonics 8, 657-663 (2014).

[16] Zang X. F. et al., Ultra-broadband terahertz absorption by exciting the orthogonal diffraction in dumbbell-shaped gratings. Sci. Rep. 5, 8901(2015).

[17] Amin, M., Farhat, M. \& Bağcı, H. An ultra-broadband multilayered graphene absorber. Opt. Express 21, 29938-29948 (2013).

[18] Shen, X. P. et al. Triple-band terahertz metamaterial absorber: design, experiment, and physical interpretation. Appl. Phys. Lett. 101, 154102 (2012).

[19] Exter, M. V. \& Grischkowsky, D. Carrier dynamics of electrons and holes in moderately doped silicon. Phys. Rev. B 41, 12140 (1990).

[20] Moharam, M. G., Grann, E. B., Pommet, D. A. \& Gaylord, T. K. Formulation for stable and efficient implementation of the rigorous coupled-wave analysis of binary gratings. J. Opt. Soc. Am. A 12, 10681076 (1995).

[21] Smith, D. R., Vier, D. C., Koschny, T. \& Soukoulis, C. M. Electromagnetic parameter retrieval from inhomogeneous metamaterials. Phys. Rev. E 71036617 (2005).

[22] Chen, L. et al. Observation of electromagnetically induced transparency-like transmission in terahertz asymmetric waveguide-cavities systems. Opt. Lett. 38, 1379-1381 (2013).

\section{FIGURE CAPTIONS}

Figure 1 Schematic of the design strategy

(a) previous design: overlapping grating interference (blue lines) with the diffraction effect (red lines)

(b) our design: overlapping substrate interference (blue lines) with the diffraction effect (red lines)

And the structure of the sample:

(c) 3D Schematic diagram;

(d) SEM photograph.

Figure 2 Analysis on the contribution of the destructive interference to the overall reflection:

(a) The interference model of the absorber;

(b) The simulated magnitudes of the reflection and transmission coefficients for the decoupled model;

(c) The simulated phases of the reflection and transmission coefficients for the decoupled model;

(d) The calculated phase difference between $\widetilde{r}_{0}$ and $\tilde{r}_{1}$ from the decoupled model;

(e) The reflectance of the grating;

(f) The reflectance of the whole structure.

Figure 3 Analyses on the contributions of the grating to the overall reflection:

(a) The diffraction efficiencies for different orders calculated by 2D-RCWA method;

(b) The ideal and optimized impedance of the grating;

(c) The reflectance of the grating;

We highlight the frequencies where [1,0] order diffraction efficiency is greater than $[0,0]$ order diffraction efficiency in grey and where the impedance-matching conditions occurred in orange.

(d)-(f) The electric field distribution with diffraction directions

(white arrows) of $\mathrm{x}-\mathrm{z}$ plane at

(d) $\mathrm{f}=1.15 \mathrm{THz}$; (e) $\mathrm{f}=1.54 \mathrm{THz}$; (f) $\mathrm{f}=1.95 \mathrm{THz}$.
Figure 4 The simulated results via CST microwave studio ${ }^{\circledR}$ (a) the absorption spectrum;

(b)-(f) the energy density distributions in the $\mathrm{x}-\mathrm{z}$ plane at $\mathrm{y}=$ $36.5 \mu \mathrm{m}$ at five absorption peaks.

Figure 5 The comparisons of the experimental results, errors and the simulated results in (a) TE mode (b) TM mode;

The dependences of the absorption spectra on incident angles in (c) TE mode (d) TM mode. 


\section{BIOGRAPHY}

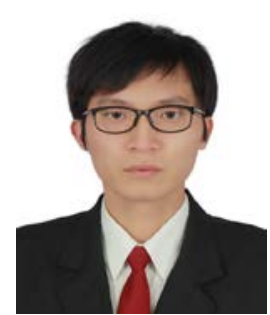

Cheng Shi received the B.S. and M.S. degree in engineering from University of Shanghai for Science and Technology, Shanghai, China, in 2012 and 2015. He is now working on his Ph.D. degree in University of Exeter, Exeter, United Kingdom. He participated in many Chinese and European projects on terahertz functional metamaterial devices and their applications His research interests are concentrated in terahertz metamaterial devices and transformation optics, which includes invisibility cloak, illusion media, absorbers and shift media.

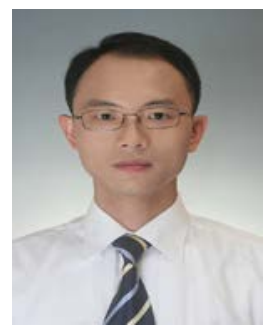

Xiaofei Zang received the B. S. degree in physics from Jiangsu normal University, Jiangsu, China, in 2005 and Ph. D degree in Electronic \& Communication Engineering, Shanghai, China, in 2012. His research interests are concentrated in the areas of Terahertz (THz) functional device and Transformation Optics. Specific research topics include $\mathrm{THz}$ absorber, $\mathrm{THz}$ isolator, cloak, high-directional antenna, and super-resolution imaging. He has published more than 30 scientific papers refereed journals.

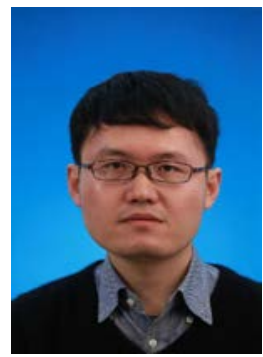

Lin Chen received the B.S. and M.S. degree from Southeast University in 2002 and 2005, both in electrical engineering, and the Ph.D. degree from Shanghai Jiao Tong University in 2008, in Optics. Now he is the associate professor in University of Shanghai for Science and Technology. His research interests include terahertz waveguide, metamaterial, and lab on chip. He has been awarded the "Chen Guang” Scholar in 2009, China Instrument Society-Jin Guofan Youth Award in 2011, and Shanghai "rising star" Scholar in 2014. He has published more than 40 SCI papers and 20 patents. As the project leader, he is also responsible for several funds from national and Shanghai.

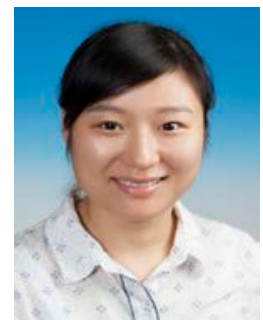

Yan Peng received her B.S. degree from the Anhui Normal University in 2004 and the Ph.D. degree from the East China Normal University in 2009, both in Physics. She is the associate professor in University for Science and Technology. Her research interests include ultrafast optics, terahertz, microstructure and high-order harmonic generation. As the project leader, Dr. Peng is responsible for National Program on Key Basic Research Project of China (973 Program, sub-project), National Development Projects of Scientific Instrument and Equipmentone National Natural Science Foundation of China, State Scholarship Fund and Shanghai Municipal Education Commission. She is awarded the "Chen Guang" Schorlar in 2012, China Instrument Society-Jin Guofan Youth Award y. Now, she has published more than 30 SCI papers and 20 patents.

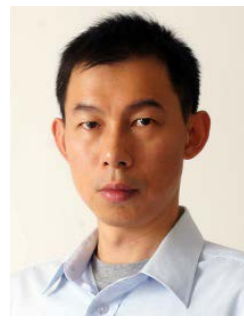

Bin Cai received the B.S. in chemistry from Nanchang University, Nanchang, China, in 1994 and the M.S. and Ph.D. degrees in optical material chemistry from Tohoku University, Japan, in 2000 and 2003, respectively. He engaged in research on organic materials for telecommunication and Terahertz applications, organic-inorganic optical nano-composites, and organic nonlinear optical materials and devices. Presently, he is with the University of Shanghai for Science and Technology.

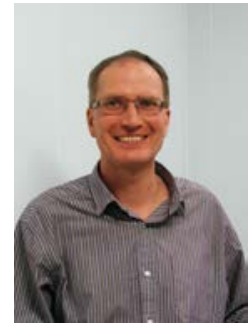

Geoff R. Nash graduated from the School of Physics, University of Bath before completing an MSc at Imperial College. For his $\mathrm{PhD}$ work, also at Bath, he was awarded the Deryk Chesterman Medal for outstanding research. He later joined QinetiQ Malvern, where we he was awarded a Royal Society Industrial Fellowship, a QinetiQ Inventors award, and became a QinetiQ Fellow. He is now Professor of Engineering Physics at the University of Exeter and his research interests include device physics, photonics, and plasmonics.. He currently holds an EPSRC Fellowship on "Disruptive Optoelectronic Manufacture using Graphene” and is Fellow of the Institute of Physics.

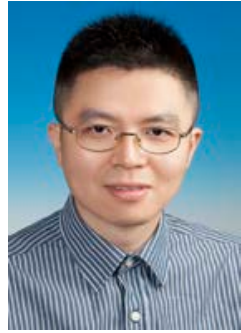

YiMing Zhu studied at Shanghai Jiaotong University from 1998 to 2004 and received B.S and M.S. degree of applied physics. He won the Japanese Government (Monbukagakusho) Scholarship in 2004 and studied in University of Tokyo studied in University of Tokyo as a doctor candidate and gained his $\mathrm{Ph}$. D. in electronics engineering department in 2008. He is now a professor in University of Shanghai for Science and Technology, the vice director of Shanghai Key Lab of Modern Optical System. Up to now, he has published more than 70 papers on SCI journals as the first author or corresponding author, including three publications on Nature Publishing Group. 


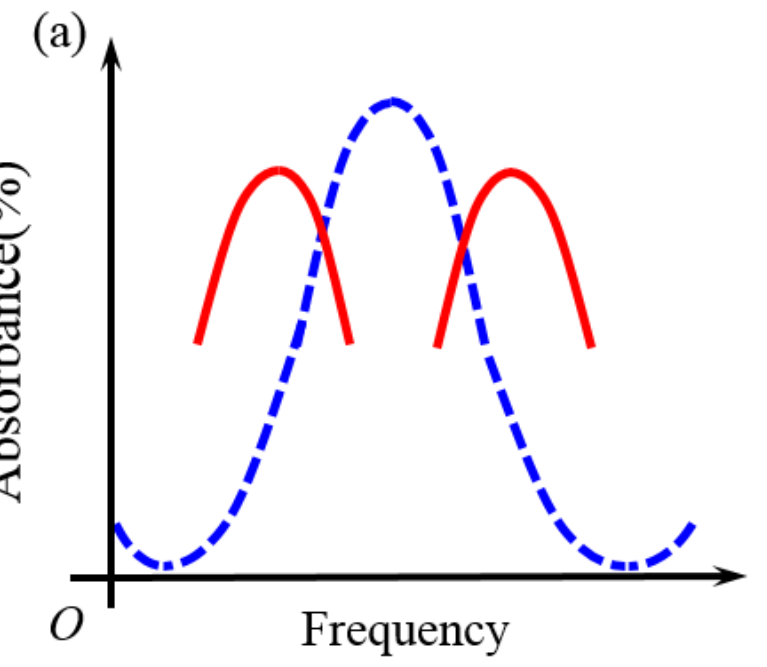

(c)

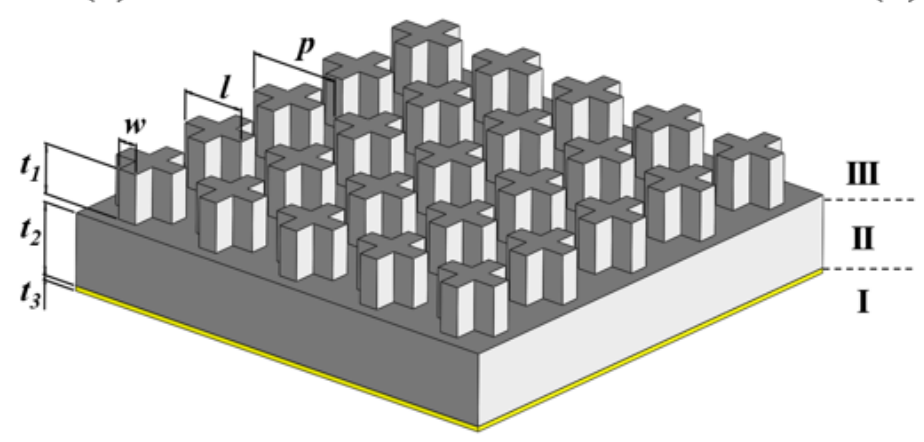

(b)

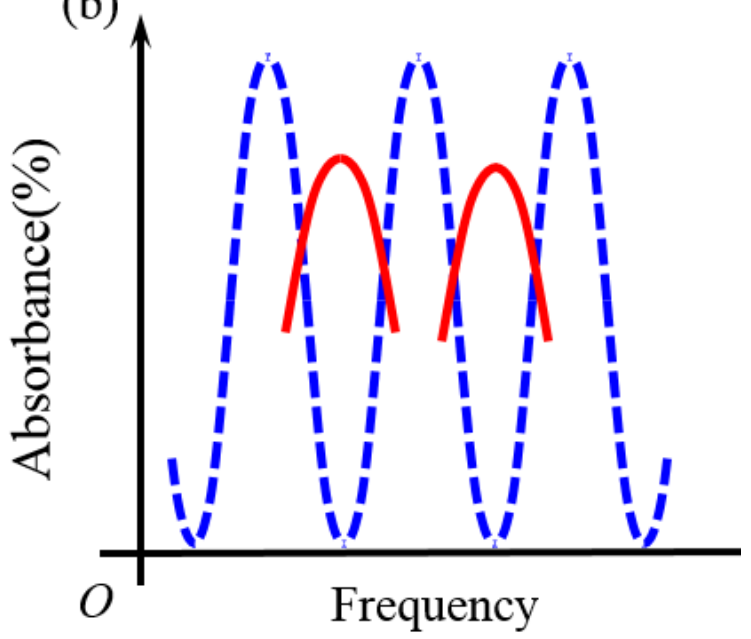

(d)

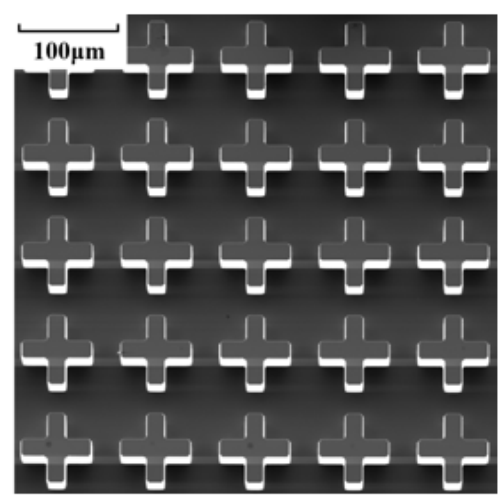

Figure 1 

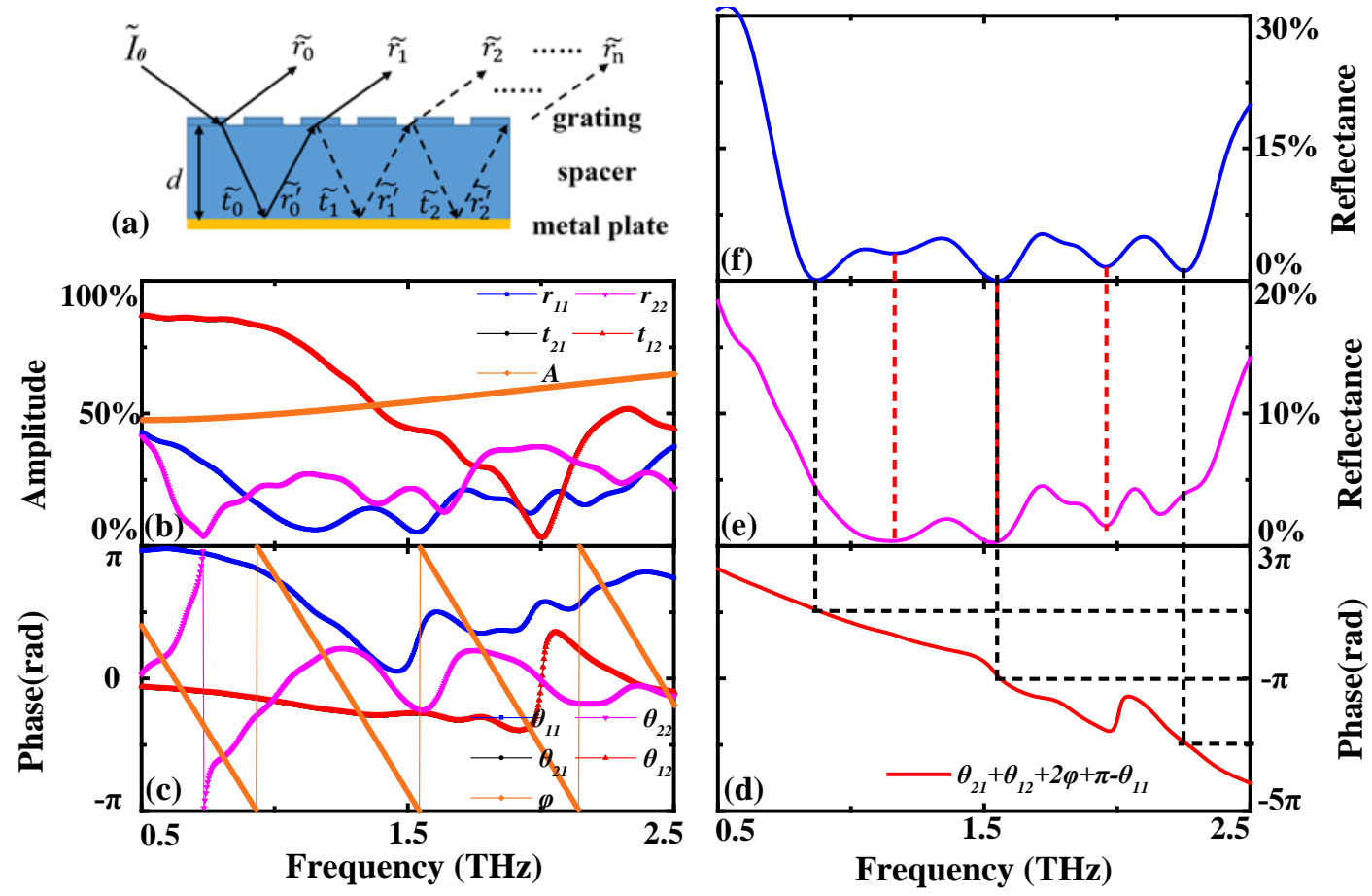

Figure 2 

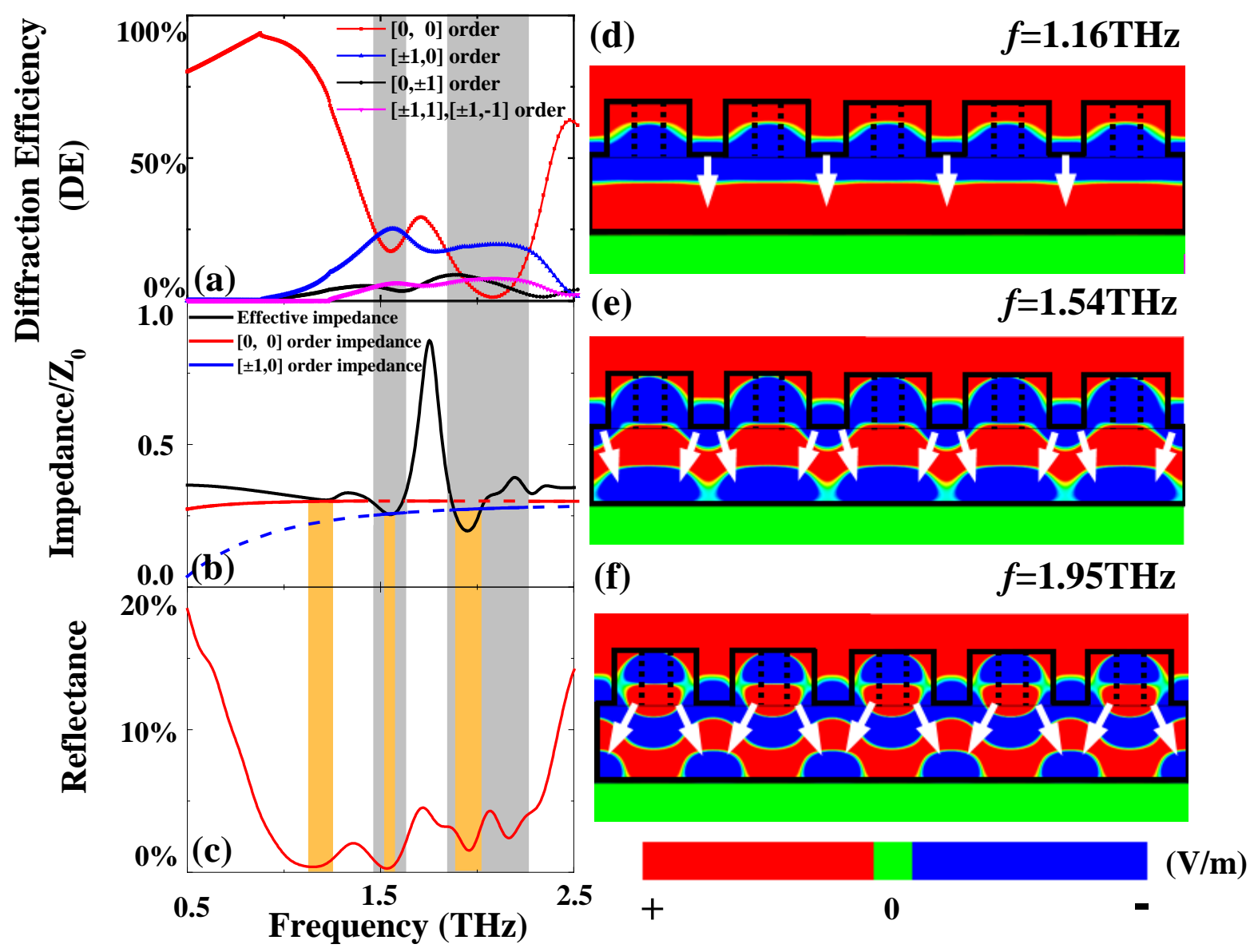

Figure 3 


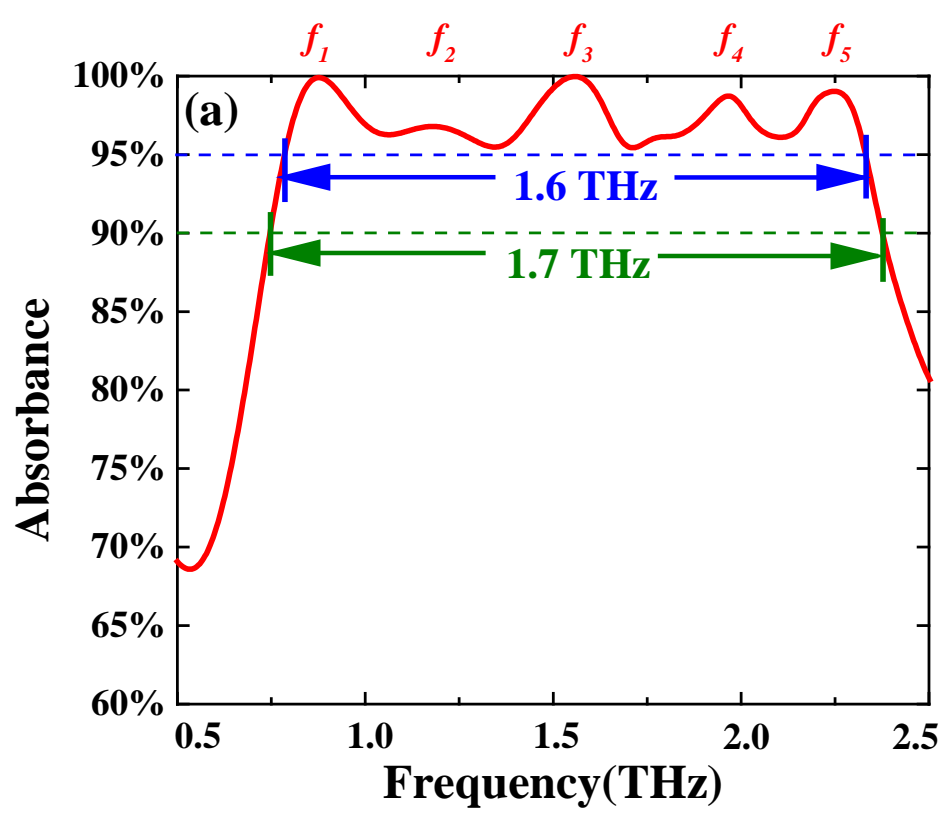

(b) $f_{1}=0.87 \mathrm{THz}$

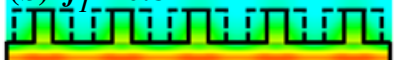

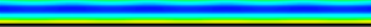

(c) $f_{2}=1.16 \mathrm{THz}$

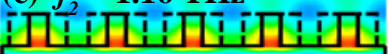

d) $f_{3}=1.54 \mathrm{THz}$

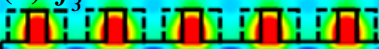

(e) $f_{4}=1.95 \mathrm{THz}$

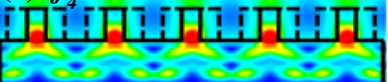

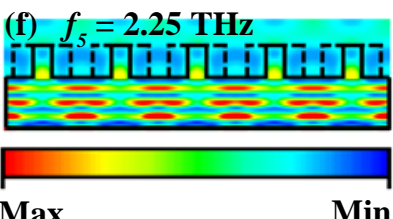

Figure 4 

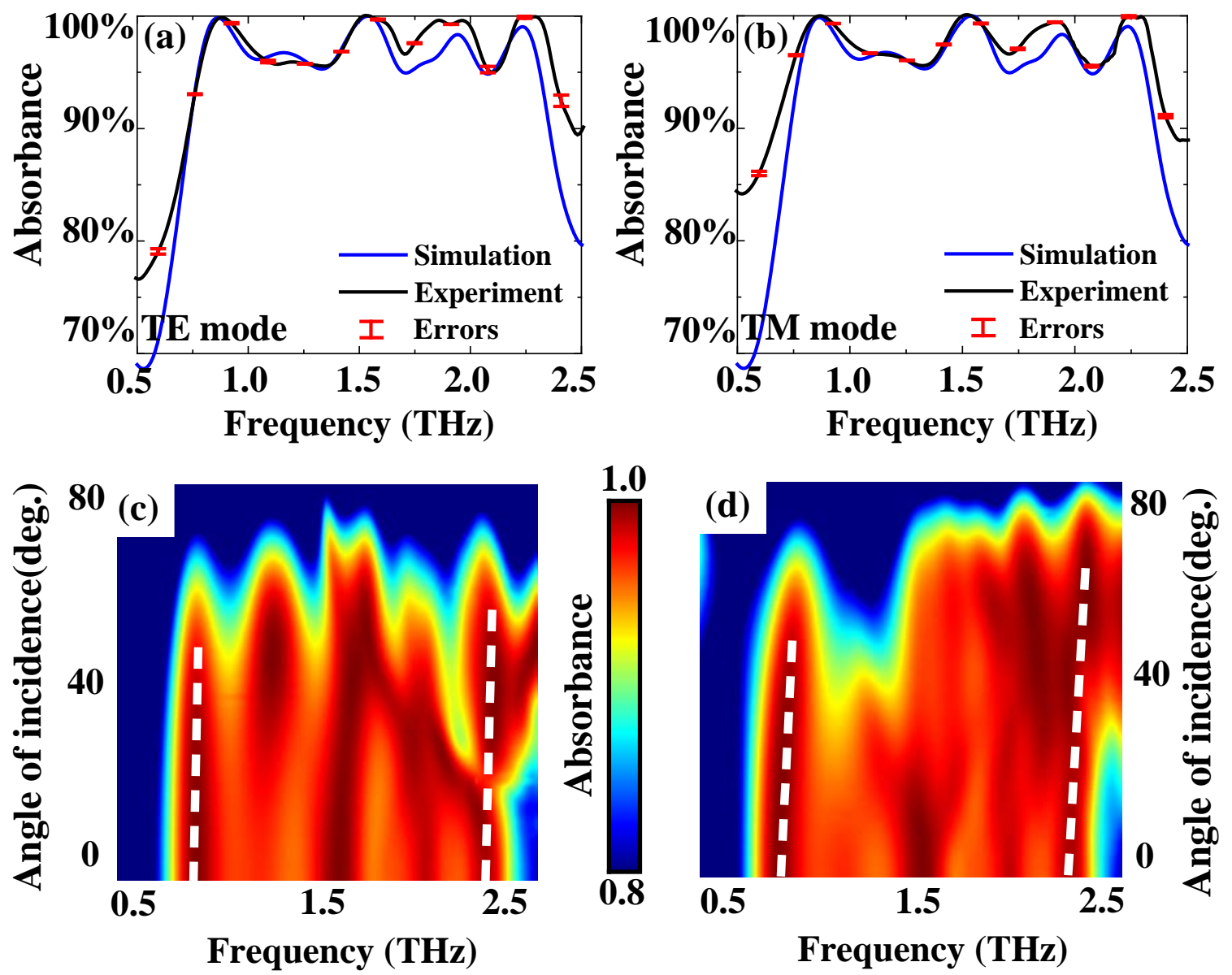

Figure 5 